\title{
Placental transfer of long-chain polyunsaturated fatty acids (LC-PUFA)
}

\author{
Berthold Koletzko ${ }^{1, *}$, Elvira Larqué ${ }^{2}$ and Hans \\ Demmelmair ${ }^{1}$ \\ ${ }^{1}$ Department of Pediatrics, Dr. von Hauner Children's \\ Hospital, Ludwig-Maximilians-University of Munich, \\ Munich, Germany \\ 2 Department of Physiology, University of Murcia, \\ Murcia, Spain
}

\begin{abstract}
Considerable evidence exists for marked beneficial effects of omega-3 long-chain polyunsaturated fatty acids (LC-PUFA) during pregnancy. The omega-3 LCPUFA docosahexaenoic acid (DHA) is incorporated in large amounts in fetal brain and other tissues during the second half of pregnancy, and several studies have provided evidence for a link between early DHA status of the mother and visual and cognitive development of her child after birth. Moreover, the supplementation of omega-3 LC-PUFA during pregnancy increases slightly infant size at birth, and significantly reduces early preterm birth before 34 weeks of gestation by $31 \%$. In our studies using stable isotope methodology in vivo, we demonstrated active and preferential materno-fetal transfer of DHA across the human placenta and found the expression of human placental fatty acid binding and transport proteins. From the correlation of DHA values with placental fatty acid transport protein 4 (FATP 4), we conclude that this protein is of key importance in mediating DHA transport across the human placenta. Given the great importance of placental DHA transport for infant outcome, further studies are needed to fully appreciate the effects and optimal strategies of omega-3 fatty acid interventions in pregnancy, dose response relationships, and the potential differences between subgroups of subjects such as women with gestational diabetes or other gestational pathology. Such studies should contribute to optimize substrate intake during pregnancy and lactation that may improve pregnancy outcome as well as fetal growth and development.
\end{abstract}

\footnotetext{
${ }^{\star}$ Corresponding author:

Berthold Koletzko, MD

Professor of Pediatrics

Dr. von Hauner Children's Hospital

Ludwig-Maximilians University of Munich

Lindwurmstr. 4, D-80337 Muenchen, Germany

Tel.: + 49-89-5160-3967

Fax: +49-89-5160-3336

E-mail: Berthold.Koletzko@med.uni-muenchen.de
}

Keywords: Fatty acid transport protein; fetus; heart fatty acid binding protein; liver fatty acid binding protein; stable isotopes.

\section{Introduction}

Evidence accumulates that the supply and metabolism of long-chain polyunsaturated fatty acids (LC-PUFA) during pregnancy is not only of importance to the pregnant woman and to the outcome of her pregnancy, but also to growth and tissue development of the fetus and to the early development of the nervous system. The LC-PUFA arachidonic (AA, 22:4 n-6) and docosahexaenoic acid (DHA, 22:6 n-3) are preferentially deposited in relatively high concentrations in developing neural cells, and they modulate the structure, fluidity and function of brain membranes [10]. The importance of brain DHA is mainly related to its significant roles in maintaining neurological and visual development. Decreased DHA contents in brain and retina lipids result in altered visual acuity, disturbance in electroretinographic measurements and learning impairment in various species [10]. AA is a major component of structural phospholipids and serves as precursor for eicosanoids, which play important roles in cell division, signal transduction and many other physiologic processes [10]. In preterm babies the availability of AA has been associated with weight at birth and growth during the first year of life [9]. In this paper we discuss the potential roles of LC-PUFA supplementation during pregnancy in perspective of preventive nutrition and their relevance for the early development of fetus and infants. The metabolism and tissue accretion of LC-PUFA in fetal brain and other tissues, the mechanisms involved in their placental transfer from mothers to their babies, and their effects on pregnancy outcomes and development of visual and other neural functions in newborns are addressed in this review.

\section{LC-PUFA supply and metabolism in the perinatal period}

LC-PUFA of the omega- 6 (n-6) and omega-3 (n-3) series are derived from the essential fatty acid precursors linoleic acid ( $n-6)$ and $\alpha$-linolenic acid $(n-3)$ by consecutive enzymatic desaturation and chain elongation (Figure 1). LC-PUFA are incorporated in practically all tissues of the fetus and infant, and they are the predominant PUFA in mammalian brain and neuronal tissues [25]. In humans, most brain LC-PUFA are accumulated during the phase 


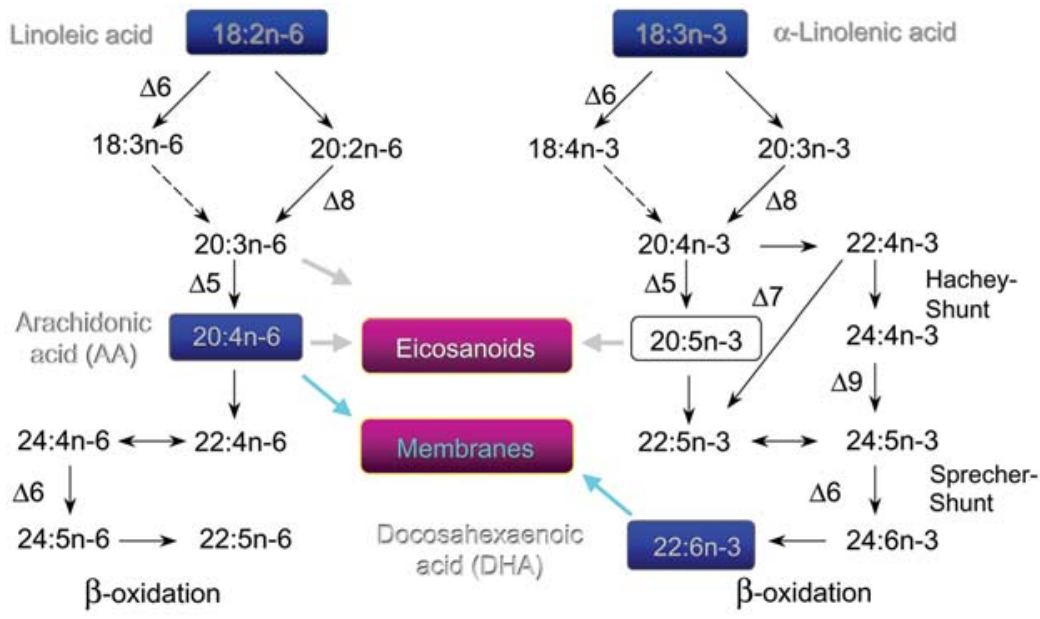

Figure 1 Metabolism of the essential fatty acids linoleic acid (18:2 n-6) and linolenic acid (18:3n-3) to long-chain polyunsaturated fatty acids (LC-PUFA).

of rapid brain growth in the last trimester of gestation and the first two years after birth. Lipids are transferred across the placenta to meet fetal demands, including the essential fatty acids linoleic (LA, C18:2 n-6) and $\alpha$-linolenic acid (ALA, C18:3 n-3), as well as LC-PUFA [10].

The fetus needs to receive appreciable amounts of preformed AA and DHA by placental transfer to meet LCPUFA accretion rates in membrane rich tissues. In adults, AA and DHA can be synthesized to a limited extent, mainly in the liver by desaturation and elongation of the essential fatty acids linoleic and $\alpha$-linolenic acid, respectively. However, the high needs in the perinatal period cannot be met by endogenous formation of LC-PUFA from the essential fatty acid precursors due to low activities of the desaturating enzymes in the fetus, placenta and in newborn infants. Thus, the fetus depends on LCPUFA provision by placental transfer [10].

The accumulation of brain DHA and AA from dietary preformed DHA and $A A$ is far more efficient than from dietary essential fatty acid precursors and their endogenous desaturation and elongation. It is estimated that about half of postnatal brain AA accretion is derived from dietary preformed $A A$ in term baboon neonates [25], while fetal plasma DHA is about 8-20-fold more effective as a substrate for brain DHA accretion in fetal baboons compared with ALA [22]. In humans the fetal and infant brain DHA content is relatively more affected by the diet than $A A$ content, suggesting that endogenous metabolic regulation of $A A$ contents is more effective [14]. Fetal tissue contents of LC-PUFA depend on maternal intake and on adequate placental transport.

\section{Placental transfer of fatty acids}

Numerous studies reported that LC-PUFA contents in cord plasma lipids are higher than in maternal plasma lipids at the time of birth [1], while contents of the parent essential fatty acids (EFA) are lower in infant than in maternal plasma lipids. These observations point to a preferential transfer of these fatty acids by the placenta. Recently, we demonstrated active materno-fetal transfer of LCPUFA using stable isotope techniques. Fatty acids labeled with the natural carbon variant ${ }^{13} \mathrm{C}$ were administrated $4 \mathrm{~h}$ before elective cesarean section to pregnant women (Figure 2). Analysis of placental tissue at the time of birth indicated preferential incorporation of DHA relative to linoleic acid, oleic acid and palmitic acid, thus, demonstrating for the first time a preferential maternofetal DHA transfer across the human placenta in vivo (Figure 3) [11].

The placental transfer of fatty acids is considered a complex process, which involves binding to membrane proteins and cytoplasmatic transport proteins [5]. It is known that placental uptake of circulating non-esterified fatty acids (NEFA) as well as NEFA released from maternal circulating triglycerides by placental lipoprotein lipase; in addition, human placental tissue expresses receptors to VLDL, LDL and HDL lipoproteins [16], which could provide fatty acids to the placenta for fetal transfer once they are released by phospholipase-A2 and intracellular lipases (Figure 4), although the significance of the latter mechanism is still unknown.

Unesterified fatty acids enter placental cells through passive diffusion as well as through a complex, saturable, protein-mediated mechanism. This transport mechanism seems to involve plasma membrane fatty-acid binding protein (FABPpm/GOT2), fatty acid translocase (FAT/CD36), fatty acid transport proteins (FATP), and fatty acid binding proteins (FABP) (Figure 5) [5]. Although the roles of these proteins in placental fatty acid uptake and metabolism are not yet fully understood, it has been suggested that the enrichment of LC-PUFA in the fetal circulation is achieved via a complex interaction of these 


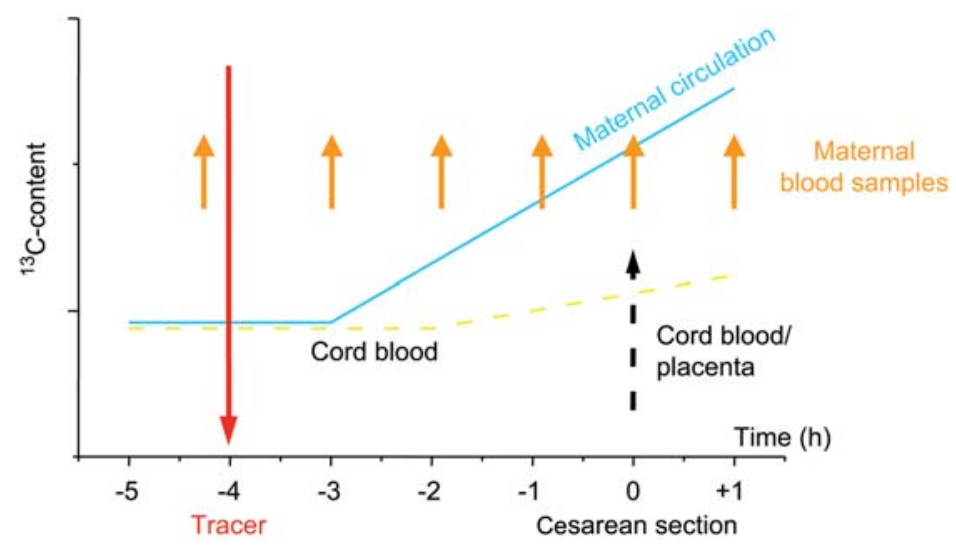

Figure 2 In vivo investigation of materno-fetal transfer of fatty acids with stable isotope techniques. Fatty acids labeled with the natural carbon variant ${ }^{13} \mathrm{C}$ are administrated $4 \mathrm{~h}$ before elective cesarean section to pregnant women. Enrichment of fatty acids is determined from maternal blood samples taken at hourly intervals, and from placental tissue and cord blood (drawn after data of [11]).

proteins. A new fatty acid binding protein located exclusively on the maternal-facing membranes named placental plasma membrane fatty acid-binding protein $\left(\mathrm{p}-\mathrm{FABP}_{\mathrm{pm}}\right)$ has been proposed to be involved in this preferential uptake of LC-PUFA by these cells. However, definitive evidence about the structure and function of p-FABPpm must await analysis of its complete amino acid and/or cDNA sequence.

In the cytosol, fatty acids are esterified within the lipid fractions of the placenta or cross the tissue in either direction bound to cytosolic proteins [5]. A family of proteins named fatty-acid binding proteins (FABP) are involved in the transfer to the fetal circulation. Two forms of FABP have been identified up to the present time in

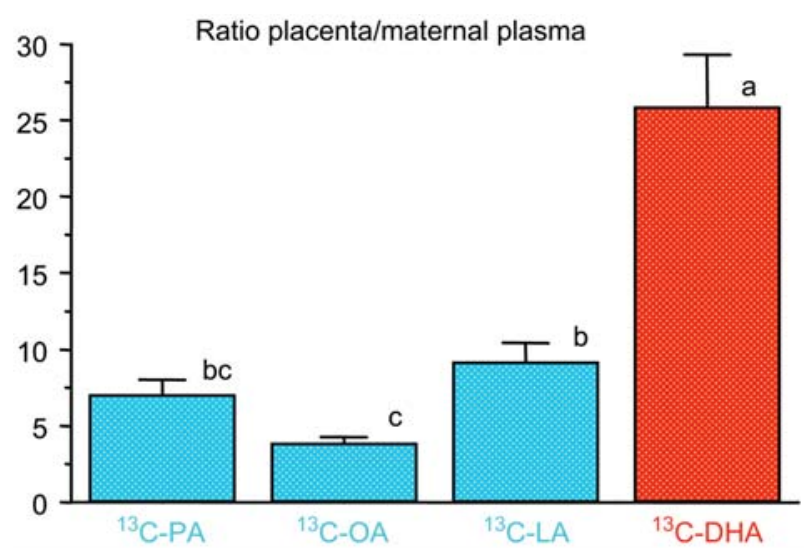

Figure 3 Results of in vivo investigation of materno-fetal transfer of ${ }^{13} \mathrm{C}$-labeled fatty acids demonstrate preferential transfer of the omega-3 long-chain polyunsaturated fatty acid DHA (docosahexaenoic acid). The ratio between labeled fatty acids (fatty acid concentration in $[\mu \mathrm{mol} / \mathrm{g}] \times$ atom percent enrichment) in placenta and maternal plasma $4 \mathrm{~h}$ after tracer administration is similar for saturated (palmitic acid, PA), monounstaurated (oleic acid, OA) and precursor essential fatty acids (linoliec acid, LA), but markedly higher for DHA (drawn after data of [11]). placenta: heart-FABP (H-FABP) and liver-FABP (L-FABP) (Figure 5) [5]. However, the mRNA expression of other cytosolic FABPs described in other tissues might occur in placental tissue as well. This potentiality should be confirmed in trophoblast cells since placenta samples contain many cell types including a large content of blood cells.

In a double-blind randomized intervention trial supported by the European Commission, the NUHEAL study, healthy pregnant women received during the second half of gestation supplements with modified fish oil providing $500 \mathrm{mg}$ DHA per day with vitamins, or a placebo oil with vitamins. In a group of 136 study participants, the mRNA expression of the membrane proteins FATP-1 and particularly of FATP-4 in placental tissue were positively correlated with the uptake of maternal DHA into placental and cord blood phospolipids. These results point to a probably very important role of placental FATP- 1 and FATP-4 for selective materno-fetal DHA transport in humans [12]

\section{LC-PUFA accretion in complicated pregnancies}

A better understanding of the mechanisms involved in the placental transfer of LC-PUFA to the neonate is of importance in order to improve fetal DHA status not only in uncomplicated pregnancies but also in disorders associated with poor DHA status, such as gestational diabetes mellitus [24] or intrauterine growth restriction (IUGR) babies [2].

In gestational diabetes mellitus, low LC-PUFA contents were found in erythrocyte and plasma phospholipids of the babies despite normal plasma AA and DHA levels in the mothers [24]. In addition, fetuses with IUGR have a lower proportion of AA and DHA in fetal blood in comparison with maternal blood as compared to control infants who are appropriate for gestational age [2]. Thus, 


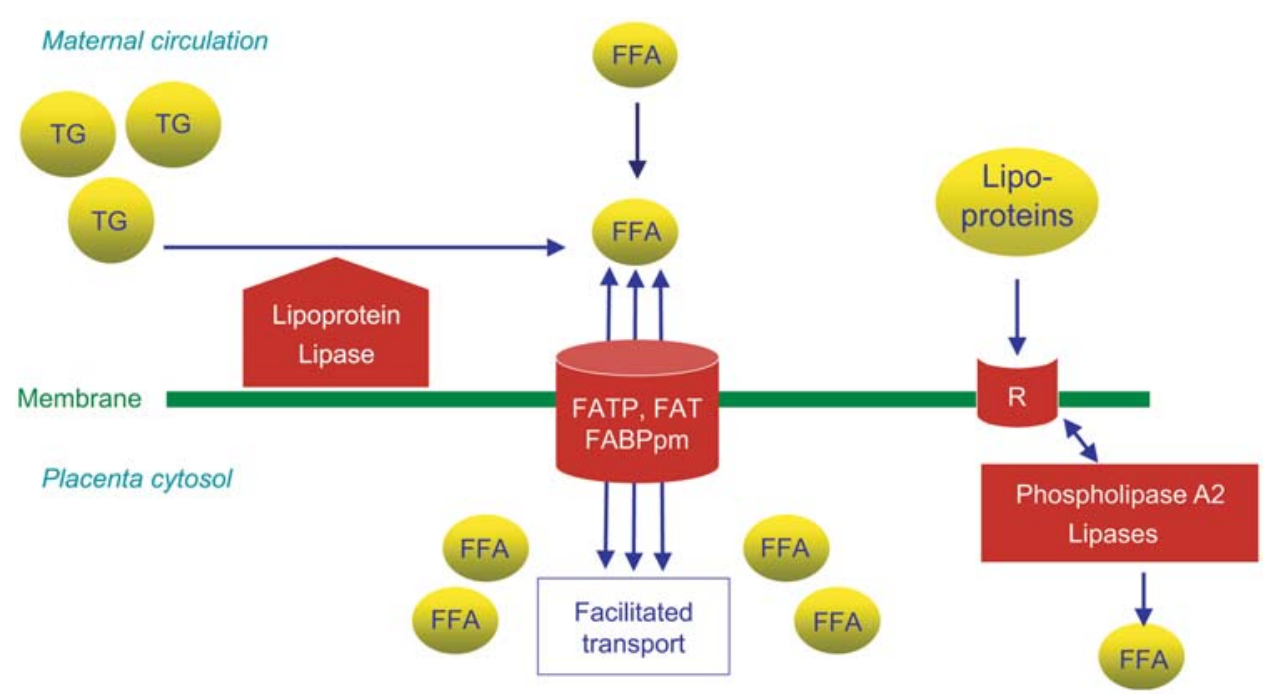

Figure 4 Placental lipid transfer. Triglycerides (TG) from TG rich lipoproteins are hydrolyzed by liporprotein lipase. Free fatty acids (FFA) may be transferred by passive diffusion as well as facilitated transport mediated by fatty acid transfer proteins (FATP), fatty acid translocase (FAT) and placental membrane fatty acid binding protein (FABPpm). In addition, recepter mediated uptake of lipoproteins and lipid release by phosholipase A2 and other lipases may provide lipids to the placenta.

a greater risk for restricted LC-PUFA supply may occur in pregnancies that are complicated with abnormal placental function affecting nutrient transfer.

The supply of preformed LC-PUFA during pregnancy or after birth could contribute to reduce the abnormalities in neurodevelopment observed in these babies. Enhanced DHA and AA formation was found in neonates with low gestational age and birth weight, suggesting that immature infants with adequate fetal growth have greater LC-PUFA formation from EFA precursors. However, such compensatory mechanism does not occur in infants with IUGR in whom the final biosynthetic pathway that leads to the synthesis of DHA is impaired [13]. Several clinical studies have shown the benefits of DHA supplementation in visual function of premature infants along with better DHA status in red blood cells and plasma [10]. Thus, targeted supplementation with LC-PUFA in pregnancies and infants with affected DHA metabolism is a major challenge to be addressed in the future.

An improved maternal n-3 LC-PUFA status by dietary intervention during pregnancy may enhance the availability of n-3 LC-PUFA to the fetus and the infant. Decsi and Koletzko [4] reviewed the human trials on the potential effects of different levels on n-3 fatty acid supply in pregnancy published from December 2002 to September 2004 and concluded that supplementation of ALA in a high dose (>10 $\mathrm{g}$ day) or DHA in a low dose (200 mg/day) did not result in significant enhancement of

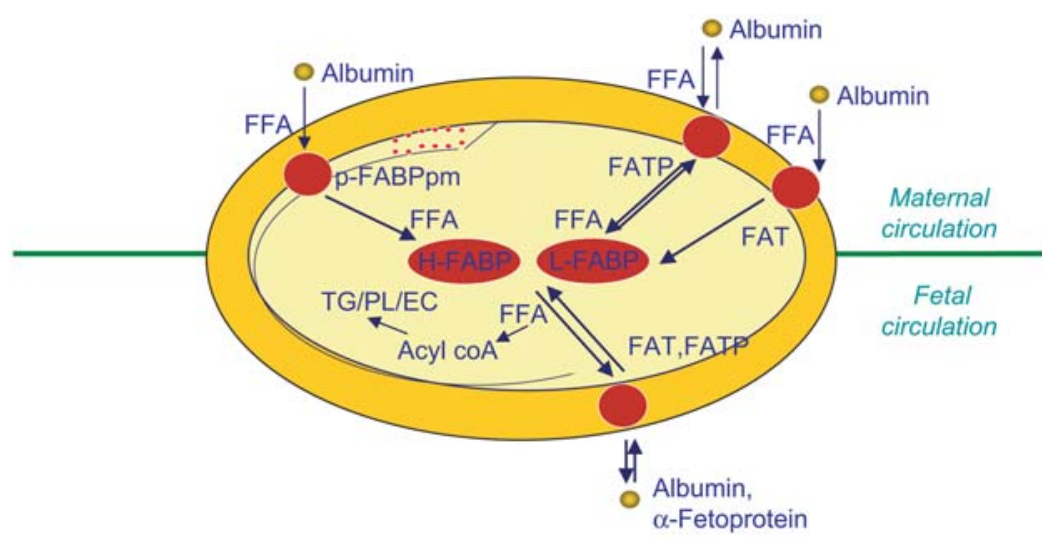

Figure 5 Placental transfer of free fatty acids (FFA) appears to be facilitated by placental fatty acid binding protein-plasma membrane (p-FABPpm), fatty acid translocase (FAT), fatty acid transport proteins (FATP), heart-fatty acid binding protein (H-FABP) and liver-fatty acid binding protein (L-FABP) (modified from a scheme suggested by Dutta Roy 2000). 
the contribution offspring. In contrast, supplementation of DHA in relatively high dose ( $>1 \mathrm{~g} /$ day) led to a significant increase in infantile DHA values in some studies.

\section{LC-PUFA supply and pregnancy outcomes}

Some, but not all studies, have shown that maternal intake of fish, fish oils and n-3 LC-PUFA result in a slightly longer duration of gestation and a somewhat higher birth weight (reviewed by Olsen, 2004 [19] and Decsi and Koletzko, 2005 [4]). It has been suggested that the effects of n-3 fatty acids on duration of pregnancy might meet a saturation point, above which no further effect on LC-PUFA n-3 can be detected, and also that they may have rapid effects, suggesting a potential use as an adjuvant to the treatment of preterm labor $[8,19]$. In the USA the cohort study "VIVA" that collected data from 2109 pregnant women, a modest decrease in fetal growth was associated with increased maternal marine n-3 PUFA consumption during pregnancy $(90 \mathrm{~g}$ between the lowest and highest quartiles of intake), with no significant changes in the length of gestation [17]. These results should be interpreted with caution, considering the limitations of observational studies which often cannot fully adjust for all confounding variables.

Recently a meta-analysis of randomized controlled intervention trials (RCT) was published reviewing effects of n-3 LC-PUFA supplementation in women with low risk pregnancies (1278 infants from 6 RCT) on pregnancy outcomes [23]. The result show that n-3 LC-PUFA supplementation during pregnancy may, to a small extent, enhance pregnancy duration and head circumference, but the mean effect is small [23]. The result of this analysis indicates that $n-3$ LC-PUFA supplementation may increase the duration of pregnancy by an average of 1.6 days. Furthermore, n-3 LC-PUFA supplementation during pregnancy was associated with a trend toward greater growth measures at birth; however, this difference was significant only for head circumference, with an average of $0.26 \mathrm{~cm}$ greater in the supplemented group, but significance was lost on sensitivity analysis [23].

A recent Cochrane review of 6 trials involving 2783 women receiving oral marine oil supplementation during pregnancy also reported a mean gestation that was 2.6 days longer than women allocated to placebo or no treatment [15]. Moreover, women in the marine oil arm had a $31 \%$ lower risk of early preterm birth before 34 completed weeks of gestation (RR $0.69,95 \% \mathrm{Cl}$ $0.49-0.99 ; 2$ trials, 860 women). Birth weight was slightly higher in infants born to women in the marine oil group compared with controls (weight mean difference $47 \mathrm{~g}$, 95\% Cl 1-93 g; 3 trials, 2440 women).

In a prospective cohort study from Denmark with 8729 pregnant women, low consumption of fish was a strong risk factor for preterm delivery and low birth weight [18].
However, no effects on the risk of preeclampsia, preterm birth, low birthweight or small gestational age was found by $\mathrm{n}-3$ LC-PUFA supplementation during pregnancy in the meta-analysis [23] or Cochrane evaluation [15].

\section{Perinatal LC-PUFA supply and infant neurodevelopment}

Whereas a large number of studies have evaluated the influence of n-3 LC-PUFA supplementation on gestational length and birth weight, only a few randomized studies examined the effects of perinatal dietary LC-PUFA on neurodevelopment.

Helland et al. performed a double blind randomized study in pregnant women receiving, from the $17^{\text {th }}-19^{\text {th }}$ week of gestation until three months after delivery, $1 \mathrm{~g}$ DHA daily provided with $10 \mathrm{~mL}$ cod liver oil, or a placebo oil (corn oil). Neonates with mature electroencephalography (EEG) test on the second day of life had a higher concentration of DHA in umbilical plasma phospholipids than neonates with immature EEG, in agreement with a relationship between DHA concentration and cerebral maturation of the newborn [6]. However, no differences were reported on EEG scores at three months of age or the Fagan test (an indicator of infants' cognitive function) at 6 and 9 months of age in the babies [6]. In contrast, at the age of four years, children who were born to mothers who had taken cod liver oil (n-3 LC-PUFA) during pregnancy and lactation had significantly higher IQ values, as assessed with the Kaufman Assessment Battery for children (K-ABC) intelligence test [7]. In addition, Colombo et al. [3] reported in a longitudinally study, showing that infants whose mothers had higher levels of DHA in red blood cells at birth, had more mature developmental profiles on single-object attention measures and more optimal performance on distractibility assessments during the first two years of life [3].

The current results show associations between early DHA status and cognitive function in infancy and early childhood. The participants of a recent expert workshop on dietary fat intake during the perinatal period held with support of the European Commission's Directorate General Research recommended that pregnant and lactating women should aim to achieving a dietary intake of $n-3$ LC-PUFA that supplies a DHA intake of at least $200 \mathrm{mg}$ per day. Intakes up to $1 \mathrm{~g} /$ day of DHA or $2.7 \mathrm{~g} /$ day of n-3 LC-PUFA have been used in randomized trials without occurrence of significant effects.

\section{Conclusions}

The potential of the early diet for modulation of the normal trajectory of brain development is of great interest. Results of recent studies show an active and preferential materno-fetal transfer of DHA across the placenta, 
apparently mediated by specific binding and transfer proteins. Fish oil supplementation during pregnancy slightly increases infant size at birth and significantly reduces early preterm birth before 34 weeks of gestation. Several studies provided evidence for a link between early DHA status in the mother and cognitive development of her child. Given the great importance of these findings, further studies are necessary to fully appreciate the effects and optimal strategies of omega-3 fatty acid interventions in pregnancy, dose response relationships, and the potential differences between subgroups of subjects such as women with gestational diabetes or other gestational abnormalities. Such studies should contribute to achieving optimized substrate intakes during pregnancy and lactation that support improved pregnancy outcomes and fetal growth and development.

\section{Acknowledgements}

The work reported herein has been carried out with partial financial support from the Commission of the European Communities, specific RTD Programme "Quality of Life and Management of Living Resources", within the $5^{\text {th }}$ Framework Programme, research grants no. QLRT-2001-00389 and QLK1-CT-200230582, and the $6^{\text {th }}$ Framework Programme, contract no. 007036 "The early nutrition programming project" (www.metabolicprogramming.org). This manuscript does not necessarily reflect the views of the Commission and in no way anticipates the future policy in this area. Additional support from the Child Health Foundation is gratefully acknowledged. BK is the recipient of a Freedom to Discover Award of the Bristol Myers Squibb Foundation, New York, NY, USA.

\section{References}

[1] Berghaus T, H Demmelmair, B Koletzko: Essential fatty acids and their long-chain polyunsaturated metabolites in maternal and cord plasma triglycerides during late gestation. Biol Neonate 77 (2000) 96

[2] Cetin I, N Giovannini, G Alvino, C Agostoni, E Riva, M Giovannini, et al.: Intrauterine growth restriction is associated with changes in polyunsaturated fatty acid fetal-maternal relationships. Pediatr Res 52 (2002) 750

[3] Colombo J, KN Kannass, DJ Shaddy, S Kundurthi, JM Maikranz, CJ Anderson, et al.: Maternal DHA and the development of attention in infancy and toddlerhood. Child Dev 75 (2004) 1254

[4] Decsi T, B Koletzko: N-3 fatty acids and pregnancy outcomes. Curr Opin Clin Nutr Metab Care 8 (2005) 161

[5] Dutta-Roy AK: Transport mechanisms for long-chain polyunsaturated fatty acids in the human placenta. Am J Clin Nutr 71 (2000) 315

[6] Helland IB, OD Saugstad, L Smith, K Saarem, K Solvoll, T Ganes, et al.: Similar effects on infants of $n-3$ and $n-6$ fatty acids supplementation to pregnant and lactating women. Pediatrics 108 (2001) 82

[7] Helland IB, L Smith, K Saarem, OD Saugstad, CA Drevon: Maternal supplementation with very-long-chain n-3 fatty acids during pregnancy and lactation augments children's IQ at 4 years of age. Pediatrics 111 (2003) 39
[8] Knudsen V, HS Hansen, ML Osterdal, TB Mikkelsen, H Mu SF Olsen: Fish oil in various doses or flax oil in pregnancy and timing of spontaneous delivery: a randomised controlled trial. Br J Obstet Gynecol 113 (2006) 536

[9] Koletzko B, M Braun: Arachidonic acid and early human growth: is there a relation? Ann Nutr Metab 35 (1991) 128

[10] Larqué E, H Demmelmair, B Koletzko: Perinatal supply and metabolism of long-chain polyunsaturated fatty acids: importance for the early development of the nervous system. In: Klimes I, Sebökova E, Howard BV, Ravussin E (eds). Lipids and insulin resistance. The role of fatty acid metabolism and fuel partitioning. Annals New York Acad Science 967 (2002) 299

[11] Larqué E, H Demmelmair, B Berger, U Hasbargen, B Koletzko: In vivo investigation of the placental transfer of ${ }^{13} \mathrm{C}$-labeled fatty acids in humans. J Lipid Res 44 (2003) 49

[12] Larqué E, S Krauss-Etschmann, C Campoy, D Hartl, J Linde, M Klingler, et al.: Docosahexaenoic acid supply in pregnancy affects placental expression of fatty acid transport proteins. Am J Clin Nutr 84 (2006) 853

[13] Llanos A, A Llanos, Y Lin, P Mena, N Salem Jr, R Uauy: Infants with intrauterine growth restriction have impaired formation of docosahexaenoic acid in early neonatal life: a stable isotope study. Pediatr Res 58 (2005) 735

[14] Makrides M, MA Neumann, RW Byard, K Simmer, RA Gibson: Fatty acid composition of brain, retina, and erythrocytes in breast- and formula-fed infants. Am J Clin Nutr 60 (1994) 189

[15] Makrides M, L Duley, SF Olsen: Marine oil, and other prostaglandin precursor, supplementation for pregnancy uncomplicated by pre-eclampsia or intrauterine growth restriction. Cochrane Database Syst Rev 3 (2006) CD003402

[16] Naoum HG, RC De Chazal, BM Eaton, SF Contractor: Characterization and specificity of lipoprotein binding to term human placental membranes. Biochim Biophys Acta 902 (1987) 193

[17] Oken E, KP Kleinman, SF Olsen, JW Rich-Edwards, MW Gillman: Associations of seafood and elongated n-3 fatty acid intake with fetal growth and length of gestation: results from a US pregnancy cohort. Am J Epidemiol 160 (2004) 774

[18] Olsen SF, NJ Secher: Low consumption of seafood in early pregnancy as a risk factor for preterm delivery: prospective cohort study. Br Med J 324 (2002) 447

[19] Olsen SF, SF Isen: Is supplementation with marine omega3 fatty acids during pregnancy a useful tool in the prevention of preterm birth? Clin Obstet Gynecol 47 (2004) 768 discussion 881

[20] Simopoulos AP, A Leaf, N Salem Jr: Essentiality of and recommended dietary intakes for omega- 6 and omega-3 fatty acids. Ann Nutr Metab 43 (1999) 127

[21] Simopoulos AP, A Leaf, N Salem Jr: Workshop on the essentiality of and recommended dietary intakes for omega-6 and omega-3 fatty acids. J Am Coll Nutr 18 (1999) 487

[22] Su H, HM Su, MC Huang, NM Saad, PW Nathanielsz, JT Brenna: Fetal baboons convert 18:3n-3-22:6n-3 in vivo. A stable isotope tracer study. J Lipid Res 42 (2001) 581

[23] Szajewska H, A Horvath, B Koletzko: Effect of n-3 longchain polyunsaturated fatty acid supplementation of women with low-risk pregnancies on pregnancy outcomes and growth measures at birth: a meta-analysis of randomized controlled trials. Am J Clin Nutr 83 (2006) 1337 
[24] Wijendran V, RB Bendel, SC Couch, EH Philipson, S Cheruku, CJ Lammi-Keefe: Fetal erythrocyte phospholipid polyunsaturated fatty acids are altered in pregnancy complicated with gestational diabetes mellitus. Lipids 35 (2000) 927
[25] Wijendran V, MC Huang, GY Diau, G Boehm, PW Nathanielsz, JT Brenna: Efficacy of dietary arachidonic acid provided as triglyceride or phospholipid as substrates for brain arachidonic acid accretion in baboon neonates. Pediatr Res 51 (2002) 265 